\title{
Editorial: reproducibilidad, replicación e investigación de implementación
}

Hemos llegado al segundo número del trigésimo aniversario de la revista Educación Matemática. Es un número festivo en más de un sentido: no sólo porque continúa con la dinámica de incluir artículos invitados con motivo del aniversario de la revista; también es festivo porque aparece en un momento en el que se ha anunciado que un colaborador de la revista, Salvador Llinares, será galardonado con la Medalla Luis Santaló que otorga el Comité Interamericano de Educación Matemática (CIAEM) (ver https://goo.gl/PFFChD), ienhorabuena Salvador!

Este número está integrado por seis artículos de investigación y un ensayo, incluye también un par de artículos invitados escritos por dos destacados investigadores en el campo de la educación matemática: Michèle Artigue y Abraham Arcavi.

El artículo de Michèle Artigue me llama particularmente la atención porque se relaciona con tópicos fundamentales para el desarrollo de la disciplina como lo son la replicación, el diseño y la implementación de innovaciones didácticas. El artículo de Artigue versa sobre la investigación de la reproducibilidad de situaciones didácticas, que ella misma impulsó en la década de los ochenta. A pesar de ser un tema de investigación que podría considerarse «viejo» y no central en el discurso actual de la investigación en educación matemática, el artículo evidencia cómo el estudio de la reproducibilidad puede estar relacionado 
con temas actuales y vigentes como evidence-based education, design-based research o task design.

Un problema fundamental en la investigación de la reproducibilidad de situaciones didácticas, es determinar las condiciones que permiten (o impiden) que una situación didáctica o diseño didáctico sea reproducible -en términos de sus efectos didácticos-, en distintos escenarios de instrucción. Así, el fenómeno de la reproducibilidad se encuentra íntimamente ligado con la práctica de la replicación, en este caso replicación de situaciones didácticas.

Pienso que el potencial de la replicación en la investigación en educación matemática ha sido subexplotado. Su estudio no sólo nos puede ayudar a identificar los facilitadores y obstáculos para reproducir y hacer robustos los diseños didácticos que generamos, sino también nos puede dar información sobre la posible solidez y generalización de los resultados de investigación que producimos (Education Committee of the European Mathematics Society, 2011), y de los métodos que utilizamos. Sin embargo, varios autores han expresado que a pesar de los beneficios que podría traer al campo de la educación matemática, los estudios de replicación no han recibido la atención debida; como lo afirma Burkhardt (2013): "Replication, a key element in scientific research, is simply not sexy." (p. 225).

La lectura del artículo de Michèle Artigue sobre reproducibilidad, nos lleva a conectarlo con una discusión que ha estado emergiendo en los últimos meses en nuestra disciplina: la investigación sobre implementación de resultados de investigación (Cai et al., 2017; Jankvist, Aguilar, Ärlebäck \& Wæge, 2017)

Así como en los estudios de reproducibilidad, en los que se trata de identificar y conceptualizar las variables que influyen en la implementación y reproducción de un diseño didáctico al nivel del salón de clases, de la misma manera podríamos intentar conceptualizar los factores que influyen en la implementación, diseminación y reproducción de innovaciones en general, que sean producidas en el campo de la educación matemática.

Estoy entendiendo la noción de innovaciones en el sentido de Century \& Cassata (2016): como un constructo general que puede abarcar no sólo diseños didácticos para el aula, sino también programas, intervenciones, procesos, aproximaciones, métodos, estrategias, tecnologías o incluso políticas educativas. Un ejemplo de implementación de una innovación se encuentra en el otro artículo invitado escrito por Abraham Arcavi, en el que se propone la implementación de una visión integradora de la enseñanza y el aprendizaje de las matemáticas. Sin embargo, el autor reconoce la complejidad que conlleva dicha implementación: 
No quisiera dar la impresión que implementar una visión integradora es algo fácil. No bastan ejemplos de excelentes problemas, ni prácticas modelo de aula. Es un emprendimiento continuo, necesariamente dependiente del contexto socio-cultural local, y que requiere constante monitoreo.

Pienso que algunas de las prospectivas que Artigue bosqueja en su artículo sobre el estudio del fenómeno de la reproducibilidad -en particular el uso de simulaciones computacionales-, son aplicables y relevantes para el desarrollo de la investigación de implementación en educación matemática.

El artículo invitado de Michèle Artigue me confirmó, cómo algunos estudios clásicos de la educación matemática gozan de una riqueza de constructos, nociones, métodos e ideas que tienen la vigencia y el potencial para ayudar a desarrollar e incluso ampliar las fronteras de la investigación en educación matemática.

Mario Sánchez Aguilar Editor Asociado

\section{REFERENCIAS}

Burkhardt, H. (2013). Methodological issues in research and development. In Y. Li \& J. N. Moschkovich (Eds.), Proficiency and Beliefs in Learning and Teaching Mathematics: Learning from Alan Schoenfeld and Günter Törner (pp. 203-236). Rotterdam: Sense Publishers.

Cai, J., Morris, A., Hohensee, C., Hwang, S., Robison, V. \& Hiebert, J. (2017). Making classroom implementation an integral part of research. Journal for Research in Mathematics Education, 48(4), 342-347.

Century, J. \& Cassata. A. (2016). Implementation research: Finding common ground on what, how, why, where, and who. Review of Research in Education, 40(1), 169-215.

Education Committee of the European Mathematics Society (2011). "Solid findings" in mathematics education. EMS Newsletter, 81, 46-48.

Jankvist, U.T., Aguilar, M.S., Ärlebäck, J.B. \& Wæege, K. (2017). Introduction to the papers of TWG23: Implementation of research findings in mathematics education. In T. Dooley \& G. Gueudet (Eds.), Proceedings of the Tenth Congress of the European Society for Research in Mathematics Education (pp. 3769-3775). Dublin, Ireland: DCU Institute of Education and ERME. 
